\title{
In situ titanium isotope measurements and the search for the source of nucleosynthetic anomalies in bulk chondrites
}

\author{
K. M. M. SHAW*1, M. PfeIFER1, C. D. COATH1, I. J. \\ PARKINSON1, T. ELLIOTT1 \\ 1Bristol Isotope Group, School of Earth Sciences, University \\ of Bristol, Queens Road, Bristol, BS8 1RJ, UK \\ (*Correspondence: kathryn.shaw@bristol.ac.uk)
}

Previous analyses of presolar dust from primitive meteorites present a wide range of nucleosynthetic anomalies. While the variable distribution of some observed particles can account for differences in bulk meteorite compositions for some isotope systems (e.g. s-process anomalies in $\mathrm{SiC}$ for mass independent Mo and Nd variations in chondrites), no carriers have been found that are evidently responsible for the highly distinctive bulk variations in $\varepsilon 46 \mathrm{Ti}$ and $\varepsilon 50 \mathrm{Ti}$ [1]. This is the object of our investigation.

The lack of discovery of Ti carriers may be explained if they have more labile mineralogies, which previous methods of leaching bulk meteorites to recover residual presolar dust (e.g. $\mathrm{SiC}$ ) destroyed. Methods for measuring presolar Ti are complicated by the $\mathrm{nm}$ to $\mu \mathrm{m}$ size of dust which prohibits exsitu analysis with chemical removal of Ti's isobaric interferences: Ca, Cr, V. In situ analysis of presolar dust in host meteorites would allow identification of a full suite of presolar dust [2].

Proteus, a prototype collision cell MC-ICPMS with mass pre-filter, reduces interferences during analysis. The pre-filter can be set to allow only a narrow range of masses (e.g. 42-54) to be introduced into the collision cell where ion species are reacted with $\mathrm{O}_{2}$ gas to form their oxide adducts, e.g. $\mathrm{TiO}+$ and measured in a region of the mass spectrum cleared by the mass pre-filter. By virtue of the differential reactivity of ion species with $\mathrm{O}_{2}$, isobaric interferences on $\mathrm{Ti}$ are greatly reduced; $\mathrm{Ca} / \mathrm{Ti}$ by $>99.9 \%$, V/Ti by $95.4 \%$ and $\mathrm{Cr} / \mathrm{Ti}$ by $99.4 \%$. The $\mathrm{TiO}+$ is collected simultaneously with a multi-ion counting array providing $\sim 80 \%$ precision on isotopic analyses for picogram quantities of laser ablated material.

With this method we have identified highly anomalous material within a CM2 chondrite, that is clearly resolved from terrestrial materials. These new data can help to uncover "missing" components needed to explain bulk Ti Solar System isotope trends [3].

[1] Trinquier et al. (2009) Science 324, 374. [2] Leitner \& Hoppe (2019) Nature Astro. 3, 725-729. [3] Steele \& Boehnke (2015) ApJ 802, 80. 